\title{
Comparison of the efficacy of vindesine and leurocristine for pediatric acute lymphoblastic leukemia and their effects on quality of life
}

\author{
Xiaowei Shi, Pisheng Zhang, Xiaohong Du, Lieguang Chen, Shuangyue Li, Zheng Fan
}

Department of Hematology, Yinzhou People's Hospital, Ningbo City, Zhejiang Province, China

Submitted: 29 June 2020; Accepted: 20 July 2020

Online publication: 18 April 2021

Arch Med Sci 2022

DOI: https://doi.org/10.5114/aoms/125544

Copyright $\odot 2022$ Termedia \& Banach

\begin{abstract}
Introduction: The present study aimed to compare the efficacy of vindesine with that of leurocristine for pediatric acute lymphoblastic leukemia and their effects on the quality of life.

Material and methods: We included 94 patients with pediatric acute lymphoblastic leukemia admitted to our hospital for treatment and categorized them into two groups: treatment with vindesine (group $\mathrm{A}, n=48$ ) and treatment with leurocristine (group $B ; n=48$ ). The effective rate, adverse reactions (thrombocytopenia, anemia, peripheral neurotoxic reaction, infection, nausea, and vomiting) and basic clinical conditions (medical expenses and length of hospital stay) of the patients were observed. In addition, interleukin 6 (IL-6) and tumour necrosis factor-alpha (TNF- $\alpha$ ) expression levels were determined by ELISA before and after treatment, posttreatment quality of life score was determined by the Karnofsky Performance Scale (KPS), and the 5-year overall survival was determined in both groups.

Results: Group A showed no significant difference in the total efficacy rate and 5 -year overall survival after treatment $(p>0.05)$ and had lower medical expenses, length of hospital stay, IL- 6 and TNF- $\alpha$ expression, and total incidence of adverse reactions and higher KPS scores than group B $(p<0.05)$. Conclusions: Although no significant difference was observed between vindesine and leurocristine in treating pediatric acute lymphoblastic leukemia, patients administered vindesine had fewer adverse reactions, shorter length of hospital stay, lower medical expenses, and higher quality of life than those administered leurocristine, indicating a potential association with decreased serum IL- 6 and TNF- $\alpha$ expression.
\end{abstract}

Key words: vindesine, leurocristine, pediatric acute lymphoblastic leukemia, quality of life, IL-6, TNF- $\alpha$.

\section{Introduction}

Pediatric acute lymphoblastic leukemia is the most common type of malignant tumor in children, accounting for more than $90 \%$ of all malignant tumors in children $[1,2]$. Children may show a variety of clinical manifestations such as paleness, dizziness, fatigue, fever, bleeding, and superficial lymphadenopathy [3]. Whether children with acute lymphoblastic leukemia can be cured requires specific analysis of the specific condition. Clinically, childhood acute lymphoblastic leukemia is divided into a low-risk group, a middle-risk group and a high-risk group.

\author{
Corresponding author \\ Xiaowei Shi \\ Department of Hematology \\ Yinzhou People's Hospital \\ No. 251 Baijiang East Road \\ Ningbo City 315040 \\ Zhejiang Province, China \\ Tel.: 0574-87017565 \\ E-mail: \\ xiaoweishi100@163.com
}


For children in the low-risk group and mediumrisk group, if they receive regular treatment, the therapeutic effect is very good, most children can be cured, and the cure rate exceeds $90 \%$. However, for the children in the high-risk group, due to the high degree of malignancy, it is often difficult to achieve the clinical effect of general chemotherapy [4, 5]. Therefore, focusing on a new treatment method that stops the progression of leukemia is crucial [6].

Vindesine, which is an alkaloid extracted from Catharanthus roseus [7], is important in the treatment of several types of malignant tumors [8]. It has mild experimental neurotoxicity and the capacity to inhibit tubulin polymerization [9]. Studies have revealed the effectiveness of vindesine in treating pediatric acute lymphoblastic leukemia, chronic myelocytic leukemia, esophageal cancer, and non-Hodgkin's lymphoma [10]. In contrast, leurocristine is clinically administered to treat acute leukemia and other malignant tumors [11] and is a potential serious neurotoxin [12]. Its mechanism of action has been well elucidated, and its anti-cancer activity and the possibility of its combination with other drugs have been well studied $[13,14]$. According to a study, leurocristine may be administered to patients with leukopenia and thrombocytopenia [15-17].

Chemotherapy is the standard treatment for pediatric acute lymphoblastic leukemia, and a rational treatment plan can effectively improve the survival rate in children. In the present study, we used vindesine and leurocristine for chemotherapy and compared their clinical efficacy and their effects on serum interlekin 6 (IL-6) and tumour necrosis factor-alpha (TNF- $\alpha$ ) expression levels and quality of life to provide references for the treatment of pediatric acute lymphoblastic leukemia.

\section{Material and methods}

\section{General characteristics}

A total of 94 patients with pediatric acute lymphoblastic leukemia were admitted to our hospital for treatment and were categorized into group A (treatment with vindesine, $n=48$ ) and group B (treatment with leurocristine, $n=$ 46). Group A comprised 25 males and 23 females aged $2-12$ years (mean age, $5.3 \pm 2.9$ years). In group A, 23 patients were engaged in house decoration and 25 were not, 24 were exposed to radiation and 24 were not, and 16 lived near chemical factories and 32 did not. In group B, there were 27 males and 19 females aged 3-13 years (mean age, $6.4 \pm 3.2$ years), including 15, 19, and 11 patients with and 31, 27, and 35 without house decoration, exposure to radiation, and living near chemical factories, respectively. This study was approved by the ethics committee of Yinzhou People's Hospital, and the guardians of the patients provided completed informed consent forms.

\section{Inclusion and exclusion criteria}

Patients who met the diagnostic criteria of pediatric acute lymphoblastic leukemia established by the World Health Organization [18], never received chemotherapy but could provide complete medical records, agreed to receive subsequent treatment at our hospital, and cooperated with the investigation and arrangement performed by our medical staff were included. Patients who had other concurrent tumors; cardiovascular, cerebral, vascular, and blood diseases; immune functional diseases, acute diseases, liver or kidney insufficiency, organ failure, and drug allergy were excluded.

\section{Treatment methods}

Both groups were treated in accordance with the Children's Cancer and Leukaemia Group chemotherapy plan [19] and were administered different drugs during the inductive delay period and delay reinforcement period. Group A was treated with vindesine (Chengdu Shuxi Pharmaceutical Co., Ltd., product No. H20057028) intravenously at a dose of $3 \mathrm{mg} / \mathrm{m}^{2}$ once per week, and group B was treated with leurocristine (Shenzhen Wanle Pharmaceutical Co., Ltd., product No.: H44021772) intravenously at a dose of $1.5 \mathrm{mg} / \mathrm{m}^{2}$ once per week.

\section{Observation indexes}

The two groups were observed, and the medical expenses and length of hospital stay were recorded.

For determination of serum IL- 6 and TNF- $\alpha$ concentrations, $5 \mathrm{ml}$ of blood was drawn 1 day before and $24 \mathrm{~h}$ after administration in a fasting condition, centrifuged for $10 \mathrm{~min}$ at $1500 \mathrm{r} / \mathrm{min}$, and placed in a refrigerator at $-70^{\circ} \mathrm{C}$ until use. Serum concentrations were measured by ELISA following the manufacturer's instructions for IL-6 (Wuhan Elabscience Biotechnology Co., Ltd., product No.: E-EL-H0102c) and TNF- $\alpha$ (Wuhan Elabscience Biotechnology Co., Ltd., product No.: E-EL-H0109c). Standard, sample, and blank control wells (without sample and ELISA reagent) were set up. Approximately $50 \mu \mathrm{l}$ of standard product was added to the standard wells and $40 \mu \mathrm{l}$ of sample diluent into the sample wells, followed by $10 \mu \mathrm{l}$ of sample (finally diluted five times). The wells were sealed and incubated at $37^{\circ} \mathrm{C}$ for $30 \mathrm{~min}$; the liquid in each well was discarded, and the wells were dried by patting and were repeatedly rinsed five 
times. Next, $50 \mu$ l of ELISA reagent was added to all wells except the blank wells, and the wells were sealed and incubated at $37^{\circ} \mathrm{C}$ for $30 \mathrm{~min}$, and then $50 \mu \mathrm{l}$ of developer $\mathrm{A}$ and $50 \mu \mathrm{l}$ of developer B were added to each well, mixed, and reacted at $37^{\circ} \mathrm{C}$ for $10 \mathrm{~min}$. Finally, $50 \mu \mathrm{l}$ of stop buffer was added to each well to stop the reaction. To calculate the IL-6 and TNF- $\alpha$ concentrations, the optical density of each well was measured at $450 \mathrm{~nm}$ using a BioTek automated ELISA instrument (Beijing Anmaige Trading Co., Ltd., China).

Clinical efficacy: Disappearance of all clinical symptoms and recovery of vital signs to the normal levels indicated complete remission; in complete remission, no leukemia cells were found in the peripheral blood and the patients managed to live a normal life. No improvement in the clinical symptoms and vital signs indicated partial remission. Improvement in the clinical symptoms and vital signs indicated that the patient was stable. No significant changes in the clinical symptoms and vital signs indicated progression.

Adverse reactions (thrombocytopenia, anemia, peripheral neurotoxic reaction, infection, nausea, and vomiting) after treatment were observed and recorded.

Patients' quality of life before and after treatment was observed and scored according to the KPS scoring criteria, which defined the independent grade as a score of $>80$, at which patients can eat and dress themselves independently; the semi-dependent grade as a score of 50-70, at which patients require assistance for daily activities such as eating and dressing; and the dependent grade as a score of $<50$, at which patients always require assistance from others in daily life. A higher score indicates better physical conditions.

\section{Follow-up}

The 94 patients were followed up by calls or visits once every 3 months for 5 years. The overall survival (OS) is the period from the first postoperative day until the last follow-up or up to the patients' death.

\section{Statistical analysis}

Statistical analysis was performed using SPSS version 22.0 (IBM Corp., USA). In the case of nominal data expressed as [n (\%)], comparison studies were conducted using the $\chi^{2}$ test for intergroup comparisons. In the case of numerical data expressed as $\bar{x} \pm S D$, comparison studies were conducted using the $t$-test for intergroup comparisons. Overall survival was determined using the Kaplan-Meier method. For all statistical comparisons, significance was defined as $p<0.05$.

\section{Results}

\section{General characteristics}

No significant difference was observed between the two groups in gender, age, abnormal child-bearing history, gestational age, delivery mode, child-bearing ages of the mother and father, engagement in house decoration, exposure to radiation, living near chemical factories, frequent infection, use of antifebriles, and family history of tumors $(p>0.05$, Table I).

\section{Comparison of basic conditions after treatment between the groups}

Patients in group A reported lower medical expenses and shorter length of hospital stay than those in group B $(p<0.05$, Table II).

\section{Comparison of inflammatory factor levels before and after treatment between the groups}

There was no significant difference in IL-6 and TNF- $\alpha$ expression before treatment $(p>0.05)$, and both groups experienced a considerable decrease after treatment $(p<0.05)$, with group A demonstrating much lower results than group B $(p<0.05$, Table III).

\section{Comparison of clinical efficacy after treatment between the groups}

After treatment, group A had 35 complete remission cases (72.92\%), 10 partial remission cases $(20.83 \%), 1$ stable case $(2.08 \%)$, and 2 progress cases $(4.17 \%)$, with a total effective rate of $93.75 \%$. Group B had 25 complete remission cases (54.35\%), 15 partial remission cases (32.61\%), 3 stable cases $(6.52 \%)$, and 3 progression cases $(6.52 \%)$, with a total effective rate of $86.96 \%$ $(p>0.05$, Table IV).

\section{Comparison of adverse reactions after treatment between the groups}

Group A had a total incidence of adverse reactions of $8.33 \%$ based on one thrombocytopenia case $(2.08 \%)$, one anemia case $(2.08 \%)$, one peripheral neurotoxic reaction case $(2.08 \%)$, no infection case $(0 \%)$, and one nausea and vomiting case $(2.08 \%)$; in group $\mathrm{B}$, there were two thrombocytopenia cases (4.35\%), two anemia cases (4.35\%), three peripheral neurotoxic reaction cases (6.52\%), two infection cases (4.35\%), and three nausea and vomiting cases (6.52\%), resulting in a total incidence of adverse reactions of $26.09 \%$ $(p<0.05$, Table V). 
Xiaowei Shi, Pisheng Zhang, Xiaohong Du, Lieguang Chen, Shuangyue Li, Zheng Fan

Table I. Comparison of general clinical materials between the two groups $[n(\%)],(x \pm S D)$

\begin{tabular}{|c|c|c|c|c|}
\hline Factor & Group A $(n=48)$ & Group B $(n=46)$ & $t / \chi^{2}$ value & $P$-value \\
\hline \multicolumn{5}{|l|}{ Gender } \\
\hline Male & $25(52.08)$ & $27(58.70)$ & \multirow[t]{2}{*}{0.416} & \multirow[t]{2}{*}{0.519} \\
\hline Female & $23(47.92)$ & $19(41.30)$ & & \\
\hline Age (days) & $5.3 \pm 2.9$ & $6.4 \pm 3.2$ & 1.748 & 0.084 \\
\hline \multicolumn{5}{|l|}{ Abnormal child-bearing history } \\
\hline Yes & $15(31.25)$ & $11(23.91)$ & \multirow[t]{2}{*}{0.632} & \multirow[t]{2}{*}{0.427} \\
\hline No & $33(68.75)$ & 35 (76.09) & & \\
\hline \multicolumn{5}{|l|}{ Gestational age (weeks) } \\
\hline$<37$ & $22(45.83)$ & $16(34.78)$ & \multirow[t]{2}{*}{1.191} & \multirow[t]{2}{*}{0.275} \\
\hline$\geq 37$ & $26(54.17)$ & $30(65.22)$ & & \\
\hline \multicolumn{5}{|l|}{ Delivery mode } \\
\hline Cesarean & $25(52.08)$ & $28(60.87)$ & \multirow[t]{2}{*}{0.737} & \multirow[t]{2}{*}{0.391} \\
\hline Natural & $23(47.92)$ & $18(39.13)$ & & \\
\hline \multicolumn{5}{|l|}{ Mother's child-bearing age } \\
\hline$<30$ & $32(66.67)$ & $29(63.04)$ & \multirow[t]{2}{*}{0.135} & \multirow[t]{2}{*}{0.713} \\
\hline$\geq 30$ & $16(33.33)$ & $17(36.96)$ & & \\
\hline \multicolumn{5}{|l|}{ Father's child-bearing age } \\
\hline$<30$ & $30(62.50)$ & $34(73.91)$ & \multirow[t]{2}{*}{1.408} & \multirow[t]{2}{*}{0.235} \\
\hline$\geq 30$ & $18(37.50)$ & $12(26.09)$ & & \\
\hline \multicolumn{5}{|l|}{ House decoration } \\
\hline Yes & $23(47.92)$ & $15(32.61)$ & \multirow[t]{2}{*}{2.286} & \multirow[t]{2}{*}{0.131} \\
\hline No & $25(52.08)$ & $31(67.39)$ & & \\
\hline \multicolumn{5}{|l|}{ History of exposure to radiation } \\
\hline Yes & $24(50.00)$ & $19(41.30)$ & \multirow[t]{2}{*}{0.716} & \multirow[t]{2}{*}{0.398} \\
\hline No & $24(50.00)$ & $27(41.30)$ & & \\
\hline \multicolumn{5}{|l|}{ Chemical factory near home } \\
\hline Yes & $16(33.33)$ & $11(23.91)$ & \multirow[t]{2}{*}{1.018} & \multirow[t]{2}{*}{0.313} \\
\hline No & $32(66.67)$ & $35(76.09)$ & & \\
\hline \multicolumn{5}{|l|}{ Frequent infection } \\
\hline$<6$ times/year & $21(43.75)$ & $18(39.13)$ & 0.207 & 0.649 \\
\hline$\geq 6$ times/year & $27(56.25)$ & $28(60.87)$ & & \\
\hline Use of antifebrile & & & & \\
\hline Occasionally (< 6 times/year) & $13(27.08)$ & $15(32.61)$ & 0.343 & 0.558 \\
\hline Frequently ( $\geq 6$ times/year) & $35(72.92)$ & $31(67.39)$ & & \\
\hline Family history of tumor & & & & \\
\hline Yes & $25(52.08)$ & $28(60.87)$ & 0.737 & 0.391 \\
\hline No & $23(47.92)$ & $18(39.13)$ & & \\
\hline
\end{tabular}

Table II. Comparison of basic conditions after treatment between the two groups $(x \pm S D)$

\begin{tabular}{|lccc|}
\hline Group & $n$ & Medical expenses (RMB yuan) & Length of stay (days) \\
\hline Group A & 48 & $25436.12 \pm 6895.23$ & $22.13 \pm 5.64$ \\
\hline Group B & 46 & $35261.22 \pm 6885.59$ & $24.51 \pm 5.03$ \\
\hline$t$ & 6.911 & 2.156 \\
\hline$P$-value & $<0.001$ & 0.034 \\
\hline
\end{tabular}


Comparison of the efficacy of vindesine and leurocristine for pediatric acute lymphoblastic leukemia and their effects on quality of life

Table III. Comparison of levels of inflammatory factors before and after treatment between the two groups $(x \pm$ SD)

\begin{tabular}{|lccccc|}
\hline Group & $n$ & \multicolumn{2}{c|}{ IL-6 $(\mathrm{pg} / \mathrm{l})$} & \multicolumn{2}{c|}{ TNF- $\alpha(\mathrm{ng} / \mathrm{l})$} \\
\cline { 3 - 6 } & & Before treatment & After treatment & Before treatment & After treatment \\
\hline Group A & 48 & $12.13 \pm 1.92$ & $8.59 \pm 1.01$ & $28.45 \pm 2.21$ & $9.21 \pm 2.08$ \\
\hline Group B & 46 & $12.14 \pm 1.93$ & $9.86 \pm 1.04$ & $29.11 \pm 2.39$ & $10.98 \pm 2.11$ \\
\hline$t$ & & 0.025 & 6.006 & 1.391 & 4.095 \\
\hline$P$-value & & 0.980 & $<0.001$ & 0.168 & $<0.001$ \\
\hline
\end{tabular}

Table IV. Comparison of total effective rate after treatment between the two groups $[n(\%)]$

\begin{tabular}{|lcccccc|}
\hline Group & $n$ & $\begin{array}{c}\text { Complete } \\
\text { remission }\end{array}$ & Partial remission & Stable & Progress & $\begin{array}{c}\text { Total effective } \\
\text { rate }\end{array}$ \\
\hline Group A & 48 & $35(72.92)$ & $10(20.83)$ & $1(2.08)$ & $2(4.17)$ & $45(93.75)$ \\
\hline Group B & 46 & $25(54.35)$ & $15(32.61)$ & $3(6.52)$ & $3(6.52)$ & $40(86.96)$ \\
\hline$\chi^{2}$ & & - & - & - & - & 1.252 \\
\hline$P$-value & & - & - & - & - & 0.263 \\
\hline
\end{tabular}

Table V. Comparison of adverse reactions after treatment between the two groups [ $n(\%)]$

\begin{tabular}{|lcccc|}
\hline Type & Group A $(n=48)$ & Group B $(n=46)$ & $\chi^{2}$ value & $P$-value \\
\hline Thrombocytopenia & $1(2.08)$ & $2(4.35)$ & 0.389 & 0.532 \\
\hline Anemia & $1(2.08)$ & $2(4.35)$ & 0.389 & 0.532 \\
\hline Peripheral neurotoxic reaction & $1(2.08)$ & $3(6.52)$ & 1.136 & 0.287 \\
\hline Infection & $0(0.00)$ & $2(4.35)$ & 2132 & 0.144 \\
\hline Nausea and vomiting & $1(2.08)$ & $3(6.52)$ & 1.136 & 0.287 \\
\hline Total incidence of adverse reactions & $4(8.33)$ & $12(26.09)$ & 5.242 & 0.022 \\
\hline
\end{tabular}

\section{Comparison of Karnofsky Performance}

Scale scores before and after treatment between the groups

There was no significant difference in the quality of life before treatment $(p>0.05)$, and both groups showed improvements with treatment $(p<$ 0.05), with group A having higher KPS scores than group B $(p<0.05$, Figure 1$)$.

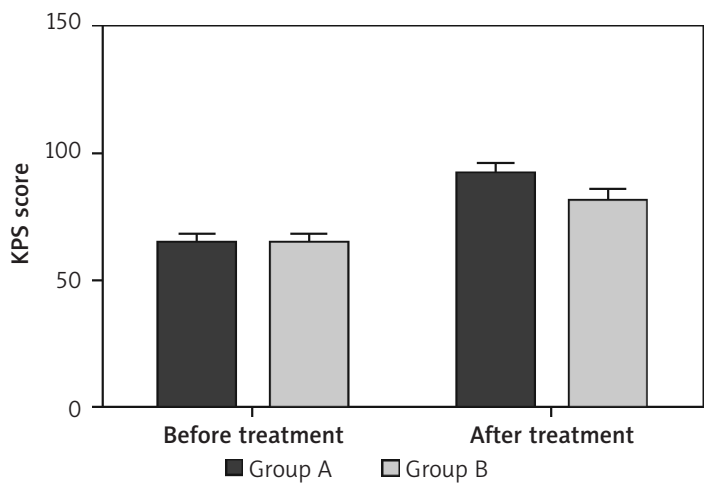

Figure 1. Comparison of Karnofsky Performance Scale (KPS) scores before and after treatment between the two groups. There was no significant difference in quality of life before treatment $(p>0.05)$, and both groups showed improvements with treatment $(p<0.05)$, with group A having higher KPS scores than group B $(p<0.05)$

$*<0.05$ as compared with group $B$ after treatment.
Five-year overall survival

According to follow-up results, the 5-year OS was $89.58 \%(5 / 43)$ in group $A$ and $82.61 \%(8 / 38)$ in group $\mathrm{B}(p>0.05$, Figure 2$)$.

\section{Discussion}

As a frequently occurring pediatric cancer [21-23], acute lymphoblastic leukemia may be caused by

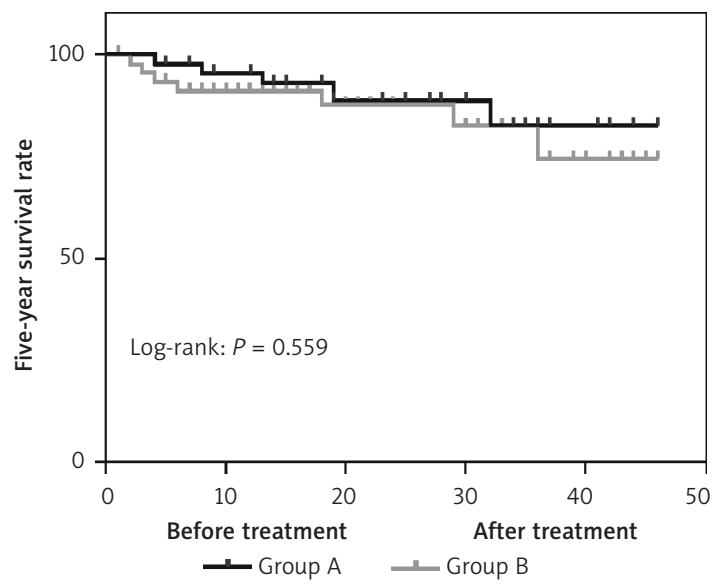

Figure 2. Comparison of 5-year overall survival (OS) after treatment between the two groups. No significant difference was observed between the two groups in the 5 -year OS after treatment $(p>0.05)$ 
genetic factors, virus infection, and immunologic deficiency. In most cases, combined chemotherapy is provided to destroy the leukemia cells in the patients [26] to achieve a higher 5-year event-free survival (EFS) [27]. However, the treatment also leads to increased toxic effects. Thus, to further eliminate the adverse effects in children, innovative methods are required [28].

In the present study, vindesine and leurocristine, both general drugs used in acute leukemia treatment, were used to treat patients with pediatric acute lymphoblastic leukemia, and significant improvements in their conditions were observed. Vindesine showed a higher vitality in the malignant tumors in the blood system and played an important role in the treatment of acute lymphoblastic leukemia, particularly in children $[29,30]$. Leurocristine is a cytotoxic alkaloid with extensive anti-tumor activity, and its efficacy depends on its concentration and continuous exposure [31]. Our results revealed no significant difference between the two groups in terms of treatment efficacy and 5-year OS, but group A had lower medical expenses and a shorter length of hospital stay than group $B$, indicating that vindesine has advantages in terms of medical expenses, length of hospital stay, and safety. Previous studies have indicated that the quality of life can be used as a major marker for the clinical treatment of acute lymphoblastic leukemia [32]. In this study, group A had a better quality of life than group B, proving vindesine's contribution to an improved quality of life after treatment compared with leurocristine.

Interleukin 6 has demonstrated extensive bioactivity in immunomodulation, tumorigenesis, hematopoiesis, and inflammation [33-35], while TNF- $\alpha$ plays a possible role in $B$ cell chronic lymphocytic leukemia [36-38]. The diagnostic value of these proinflammatory factors [39] in pediatric acute lymphoblastic leukemia has been proven in previous studies [40], and their expression in the serum of patients with leukemia often increases abnormally. The serum IL-6 and TNF- $\alpha$ expression in the two groups showed a sharp reduction after treatment, which was more significant in group A than in group B. Although this study has demonstrated the superior performance of vindesine in reducing the incidence of adverse reactions, length of hospital stay, medical expenses, and serum IL- 6 and TNF- $\alpha$ expression and improving the quality of life after treatment, it has limitations and fails to present the specific mechanisms of vindesine and leurocristine that affect the serum IL- 6 and TNF- $\alpha$ expression levels. Thus, further studies are required to verify the mechanisms of IL- 6 and TNF- $\alpha$ in pediatric acute lymphoblastic leukemia, and basic experiments with larger sample sizes should be conducted to validate our results.
In conclusion, although vindesine and leurocristine showed no significant difference in terms of efficacy and 5-year OS, the vindesine group (group A) demonstrated fewer adverse reactions, shorter length of hospital stay, lower medical expenses, and better quality of life than the leurocristine group (group B), which is possibly related to the reduced serum IL- 6 and TNF- $\alpha$ expression.

\section{Conflicts of interest}

The authors declare no conflict of interest.

\section{References}

1. Ross ME, Zhou X, Song G, et al. Classification of pediatric acute lymphoblastic leukemia by gene expression profiling. Blood 2003; 102: 2951-9.

2. Nordlund J, Bäcklin CL, Wahlberg P, et al. Genome-wide signatures of differential DNA methylation in pediatric acute lymphoblastic leukemia. Genome Biol 2013; 14: r105.

3. Gaipa G, Basso G, Biondi A, Campana D. Detection of minimal residual disease in pediatric acute lymphoblastic leukemia. Cytometry B Clin Cytom 2013; 84: 359-69.

4. Ravindranath $Y$. Recent advances in pediatric acute lymphoblastic and myeloid leukemia. Curr Opin Oncol 2003; 15: 23-35.

5. Aplenc R, Thompson J, Han P, et al. Methylenetetrahydrofolate reductase polymorphisms and therapy response in pediatric acute lymphoblastic leukemia. Cancer Res 2005; 65: 2482-7.

6. Carroll WL, Bhojwani D, Min DJ, et al. Pediatric acute lymphoblastic leukemia. Hematology Am Soc Hematol Educ Program 2003; 102-31.

7. Gralla RJ, Tan CT, Young CW. Vindesine a review of phase-II trials. Cancer Chemother Pharmacol 1979; 2: 271-4.

8. Kelsen D, Bains M, Cvitkovic E, Golbey R. Vindesine in the treatment of esophageal carcinoma: a phase II study. Cancer Treat Rep 1979; 63: 2019-21.

9. Kelsen D, Gralla R, Cheng E, Martini N. Vindesine in the treatment of malignant mesothelioma: a phase II study. Cancer Treat Rep 1983; 67: 821-2.

10. Duan YT, Sangani ChB, Liu W, Soni KV, Yao Y. New promises to cure cancer and other genetic diseases/ disorders: epi-drugs through epigenetics. Curr Top Med Chem 2019; 19: 972-94.

11. Albert DM, Wong VG, Henderson ES. Ocular complications of vincristine therapy. Arch Ophthalmol 1967; 78: 709-13.

12. Rosenthal S, Kaufman S. Vincristine neurotoxicity. Ann Intern Med 1974; 80: 733-7.

13. Silverman JA, Deitcher SR. Marqibo ${ }^{\oplus}$ (vincristine sulfate liposome injection) improves the pharmacokinetics and pharmacodynamics of vincristine. Cancer Chemother Pharmacol 2013; 71: 555-64.

14. Douer D. Efficacy and safety of vincristine sulfate liposome injection in the treatment of adult acute lymphocytic leukemia. Oncologist 2016; 21: 840-7.

15. Mora E, Smith EML, Donohoe C, Hertz DL. Vincristine-induced peripheral neuropathy in pediatric cancer patients. Am J Cancer Res 2016; 6: 2416.

16. Tay CG, Lee VWM, Ong LC, Goh KJ, Ariffin H, Fong CY. Vincristine-induced peripheral neuropathy in survivors of childhood acute lymphoblastic leukaemia. Pediatr Blood Cancer 2017; 64. 
17. Xu P, Jiang Y, Zuo H, et al. Vincristine-loaded platelets coated with anti-CD41 mAbs: a new macrophage targeting proposal for the treatment of immune thrombocytopenia. Biomater Sci 2019; 7: 4568-77.

18. Vardiman JW, Thiele J, Arber DA, et al. The 2008 revision of the World Health Organization (WHO) classification of myeloid neoplasms and acute leukemia: rationale and important changes. Blood 2009; 114: 937-51.

19. Cui L, Li ZG, Chai YH, et al. Outcome of children with new ly diagnosed acute lymphoblastic leukemia treated with CCLG-ALL 2008: The first nation-wide prospective multicenter study in China. Am J Hematol 2018; 93: 913-20.

20. Terret C, Albrand G, Moncenix G, Droz JP. Karnofsky performance scale (KPS) or physical performance test (PPT)? That is the question. Crit Rev Oncol Hematol 2011; 77: 142-7.

21. Gao RW, Dusenbery KE, Cao Q, Smith AR and Yuan J. Augmenting total body irradiation with a cranial boost before stem cell transplantation protects against post-transplant central nervous system relapse in acute lymphoblastic leukemia. Biol Blood Marrow Transplant 2018; 24: 501-6.

22. Cooper SL, Brown PA. Treatment of pediatric acute lymphoblastic leukemia. Pediatr Clin North Am 2015; 62: 61-73.

23. Davidsson J, Lilljebjörn H, Andersson A, et al. The DNA methylome of pediatric acute lymphoblastic leukemia. Hum Mol Genet 2009; 18: 4054-65.

24. Sinnett D, Krajinovic M, Labuda D. Genetic susceptibility to childhood acute lymphoblastic leukemia. Leuk Lymphoma 2000; 38: 447-62

25. Kawamata N, Ogawa S, Zimmermann M, et al. Molecular allelokaryotyping of pediatric acute lymphoblastic leukemias by high-resolution single nucleotide polymorphism oligonucleotide genomic microarray. Blood 2008; 111: 776-84

26. Rivera G, Pui $\mathrm{CH}$, Abromowitch $\mathrm{M}$, et al. Improved outcome in childhood acute lymphoblastic leukaemia with reinforced early treatment and rotational combination chemotherapy. Lancet 1991; 337: 61-6.

27. Nadeau G, Ouimet-Grennan E, Aaron M, et al. Identification of genetic variants associated with skeletal muscle function deficit in childhood acute lymphoblastic leukemia survivors. Pharmgenomics Pers Med 2019; 12: 33

28. Suárez-Llanos JP, Vallejo-Torres L, García-Bello MÁ, et al. Cost-effectiveness of the hospital nutrition screening tool CIPA. Arch Med Sci 2019; 16: 273-81.

29. Dancey J, Steward WP. The role of vindesine in oncology - recommendations after 10 years' experience. Anticancer Drugs 1995; 6: 625-36.

30. Renbarger JL, McCammack KC, Rouse CE, Hall SD. Effect of race on vincristine-associated neurotoxicity in pediatric acute lymphoblastic leukemia patients. Pediatr Blood Cancer 2008; 50: 769-71.

31. Mao W, Wu F, Lee RJ, Lu W, Wang J. Development of a stable single-vial liposomal formulation for vincristine. Int J Nanomedicine 2019; 14: 4461-74.

32. Polikandrioti M, Panoutsopoulos G, Tsami A, et al. Assessment of quality of life and anxiety in heart failure outpatients. Arch Med Sci Atheroscler Dis 2019; 4: e38-6.

33. Polikandrioti M, Kalafatakis F, Koutelekos I, Kokoularis D. Fatigue in heart failure outpatients: levels, associated factors, and the impact on quality of life. Arch Med Sci Atheroscler Dis 2019; 4: e103-12.

34. Akira S, Hirano T, Taga T, Kishimoto T. Biology of multifunctional cytokines: IL 6 and related molecules (IL 1 and TNF). FASEB J 1990; 4: 2860-7.
35. Nakajima K, Yamanaka Y, Nakae K, et al. A central role for Stat3 in IL-6-induced regulation of growth and differentiation in M1 leukemia cells. EMBO J 1996; 15: 3651-8.

36. Foa R, Massaia M, Cardona S, et al. Production of tumor necrosis factor-alpha by B-cell chronic lymphocytic leukemia cells: a possible regulatory role of TNF in the progression of the disease. Blood 1990; 76: 393-400.

37. Sethi G, Sung B, Aggarwal BB. TNF: a master switch for inflammation to cancer. Front Biosci 2008; 13: 5094-107.

38. Digel W, Stefanic M, Schoniger W, et al. Tumor necrosis factor induces proliferation of neoplastic B cells from chronic lymphocytic leukemia. Blood 1989; 73: 1242-6.

39. Xing Z, Gauldie J, Cox G, et al. IL-6 is an antiinflammatory cytokine required for controlling local or systemic acute inflammatory responses. J Clin Invest 1998; 101: 311-20.

40. Pels E. Concentration of tumour necrosis factor-alpha in saliva of patients with acute lymphoblastic leukaemia in relation to oral mucositis during chemotherapy. Chemo Open Access 2015; 4: 2. 